Southern Illinois University Edwardsville

SPARK

SIUE Faculty Research, Scholarship, and Creative Activity

6-18-2016

\title{
Experimental and Computational Study of Gas Bubble Removal in a Microfluidic System Using Nanofibrous Membranes
}

\author{
Hamed Gholami Derami \\ Ravindra Vundavilli \\ Jeff Darabi \\ jdarabi@siue.edu
}

Follow this and additional works at: http://spark.siue.edu/siue_fac

Part of the Mechanical Engineering Commons

\section{Recommended Citation}

Gholami Derami, Hamed; Vundavilli, Ravindra; and Darabi, Jeff, "Experimental and Computational Study of Gas Bubble Removal in a Microfluidic System Using Nanofibrous Membranes" (2016). SIUE Faculty Research, Scholarship, and Creative Activity. 45.

http://spark.siue.edu/siue_fac/45

This Article is brought to you for free and open access by SPARK. It has been accepted for inclusion in SIUE Faculty Research, Scholarship, and Creative Activity by an authorized administrator of SPARK. For more information, please contact gpark@siue.edu. 


\section{Cover Page Footnote}

The definitive version of this article was published by Springer in Microsystem Technologies. The final publication is available at Springer via http://dx.doi.org/10.1007/s00542-016-3020-2. 
This is an author-created, un-copyedited version of an article published in Microsystem Technologies. The final publication is available online at DOI 10.1007/s00542-016-3020-2

\title{
Experimental and Computational Study of Gas Bubble Removal in a Microfluidic System Using Nanofibrous Membranes
}

\author{
Hamed Gholami Derami, Ravindra Vundavilli, Jeff Darabi* \\ Department of Mechanical Engineering \\ Southern Illinois University Edwardsville
}

Edwardsville, IL 62026

*Corresponding author, Phone: 618-650-3382; Email: jdarabi@ siue.edu

\begin{abstract}
This paper presents a simple and efficient method for removing gas bubbles from a microfluidic system. This bubble removal system uses a $\mathrm{T}$-junction configuration to generate gas bubbles within a water-filled microchannel. The generated bubbles are then transported to a bubble removal region and vented through a hydrophobic nanofibrous membrane. Four different hydrophobic Polytetrafluorethylene (PTFE) membranes with different pore sizes ranging from 0.45 to $3 \mu \mathrm{m}$ are tested to study the effect of membrane structure on the system performance. The fluidic channel width is $500 \mu \mathrm{m}$ and channel height ranges from 100 to $300 \mu \mathrm{m}$. Additionally, a 3D computational fluid dynamics (CFD) model is developed to simulate the bubble generation and its removal from a microfluidic system. Computational results are found to be in a good agreement with the experimental data. The effects of various geometrical and flow parameters on bubble removal capability of the system are studied. Furthermore, gas-liquid two-phase flow behaviors for both the complete and partial bubble removal cases are thoroughly investigated. The results indicate that the gas bubble removal rate increases with increasing the pore size and channel height but decreases with increasing the liquid flow rate.
\end{abstract}

Keywords, Bubble removal, nanofibrous hydrophobic membrane, microfluidic system, bubble trap, T-junction, numerical simulation, volume of fluid (VOF), CFD. 
This is an author-created, un-copyedited version of an article published in Microsystem Technologies. The final publication is available online at DOI 10.1007/s00542-016-3020-2

\section{Introduction}

The field of microfluidics has advanced steadily with the development of microchannels, micropumps, lab-on-a-chip systems, DNA chips, cell culturing, bio-sensors, and micro total analysis systems [1-2]. A wide range of applications for microfluidic devices have continued to grow in recent years, and one of the common problems in these types of devices is the formation of gas bubbles in the fluidic channels. The likelihood of gas bubble formation is quite high in these systems, especially in applications where heating is required as part of the experiment [3]. In addition, bubbles can be introduced into the fluidic channels when tubing is connected to the inlet ports or porous materials such as Polydimethylsiloxane (PDMS) are used [4]. A number of experimental and computational studies have been conducted over the past decade on bubble generation, droplet formation, and bubble removal from microchannels [5-8]. The most common techniques used for bubble generation in a microchannel include a T-junction [9-12], flowfocusing devices [13-16] and other methods [17-18].

The presence of gas bubbles inside microfluidic channels is not always considered a downside to the system. There are some applications where gas bubbles play a positive role inside fluidic channels such as fluid mixing [19], echo-contrast agents [17] and microchannel reactors used in direct synthesis of hydrogen peroxide [5]. However, in many applications such as on-chip PCR [3], on-chip cell culture [20], and micro fuel cells [21], gas bubbles are problematic and have adverse effects on the system performance. Since this is one of the common issues in microfluidics devices, especially in biological applications, different bubble removal strategies have been proposed to improve the performance of microfluidic systems. Karlsson et al. [3], Skelly et al. [20], and Lochovsky et al. [22] used geometrically-enhanced bubble traps and removed gas bubbles using a PDMS substrate by applying a vacuum to one side of the substrate. Johnson et al. [23] fabricated a three-layered device and studied the effect of exposed PDMS membrane area on the bubble removal rate. Xu et al. [24] used PMMA hydrophobic porous membranes on a straight channel to remove unwanted gas bubbles and derived a correlation for extraction time. Zhu [25] employed a hydrophilic membrane in cross-flow and dead-end configurations and studied their bubble removal characteristics. Meng et al. [18] proposed two different methods for gas bubbles removal, a special pattern of venting capillaries on a hydrophobic surface and a modified venting pattern which used a hydrophobic porous membrane in the middle of convergent-divergent 
capillaries. Sung et al. [26] used some bubble barriers inside a channel with venting holes to prevent gas bubbles from moving further downstream. A de-bubbler chamber accommodating several cylindrical-shaped air-liquid interfaces was presented by Cheng et al. [27]. In this device, bubbles merged together and vented through air pillars to the atmosphere.

In addition to the aforementioned experimental studies, several numerical approaches have been proposed to study two-phase air-water flows. Some of the more popular methods include LatticeBoltzmann method [28], Level set method [29], and Volume of Fluid (VOF) method [30]. Kang et al. [31] used the VOF method and studied the bubble generation in a T-junction and its transport through a serpentine channel. Taha et al. [7, 32] employed the VOF method to study bubble dynamics in a slug flow. Qian et al. [5] extensively studied the bubble generation in a straight channel with a T-junction and investigated the effects of various parameters on bubble size using the VOF method. Fukagata et al. [33] used the level set method and studied the dynamics of gas bubbles and convective heat transfer in capillaries. Gobby et al. [34] performed a computational study on the mixing characteristics of methanol and oxygen in a T-junction configuration. Fei et al. [28] used the Lattice-Boltzmann method to investigate the bubble transport in a direct methanol fuel cell (DMFC).

This work presents a comprehensive study to investigate the effects of various parameters such as channel depth, channel width, membrane properties, and liquid flow rates on bubble extraction from a microfluidic device. A continuous stream of uniform bubbles is first generated using a Tjunction along the channels and later removed using gas permeable nanofibrous membranes. Four different hydrophobic PTFE membranes are tested and the maximum gas flow rate for the complete removal of gas slugs from the microchannel at a given water flow rate is determined. The use of hydrophobic nanofibrous membranes eliminates the need to apply vacuum on one side of the membrane, which makes the experimental setup easier to build and operate. Due to small scales of the system, bubble generation and removal occur rapidly and a set of high speed imaging equipment is required to capture detailed information about this phenomenon. Thus, in order to gain an in-depth understanding of the bubble generation and removal process, and to minimize 
fairly labor-intensive and high cost steps associated with fabrication and testing of these devices, a 3D computational model is developed in ANSYS Fluent (release 15.0). This model is then used to investigate the maximum bubble removal rate at various operating and geometric conditions. The computational domain consists of a T-junction to generate gas bubbles and a cavity on which a hydrophobic membrane is bonded parallel to the main fluidic channel to vent the gas bubbles.

\section{Materials, methods and assembly}

\subsection{Materials}

Figure 1 shows a schematic diagram of an experimental setup used in this study to generate and remove bubbles from a microfluidic device. A polyimide film was used to form fluidic channels of desired dimensions. The fluidic channels were cut in the polyimide film and sandwiched between a glass substrate and a base cover. Holes were drilled into the glass substrate to provide inlet and outlet ports for the channel. A rectangular opening was cut on the base plate and covered with a piece of PTFE membrane to extract the bubbles from the channel. Air and water were introduced into the channel using two pumps. A syringe pump was used to inject liquid into the microfluidic device and a bidirectional pump was used to pump the gas phase. A T-junction was used to generate uniform bubbles in the fluidic channel. Four different hydrophobic nanofibrous membranes were tested and their performances were characterized for bubble extraction from the microfluidic device. The specifications of these membranes are summarized in Table 1.

As depicted in Figure 2, atomic force microscope (AFM) images of these PTFE membranes show that they all have nanofibrous structures with highly interconnected pores. The sizes of these interconnected pores are larger than the diameter of the interconnecting fibers [35]. Sterlitech's PTFE membranes are laminated onto a polypropylene support, while Emflon PTFE membrane (Pall Corporation, NY, USA) is spun bonded on non-woven polyester support. Advantec's PTFE membrane is a thin, highly porous unsupported membrane for air and gas venting applications. 
This is an author-created, un-copyedited version of an article published in Microsystem Technologies. The final publication is available online at DOI 10.1007/s00542-016-3020-2

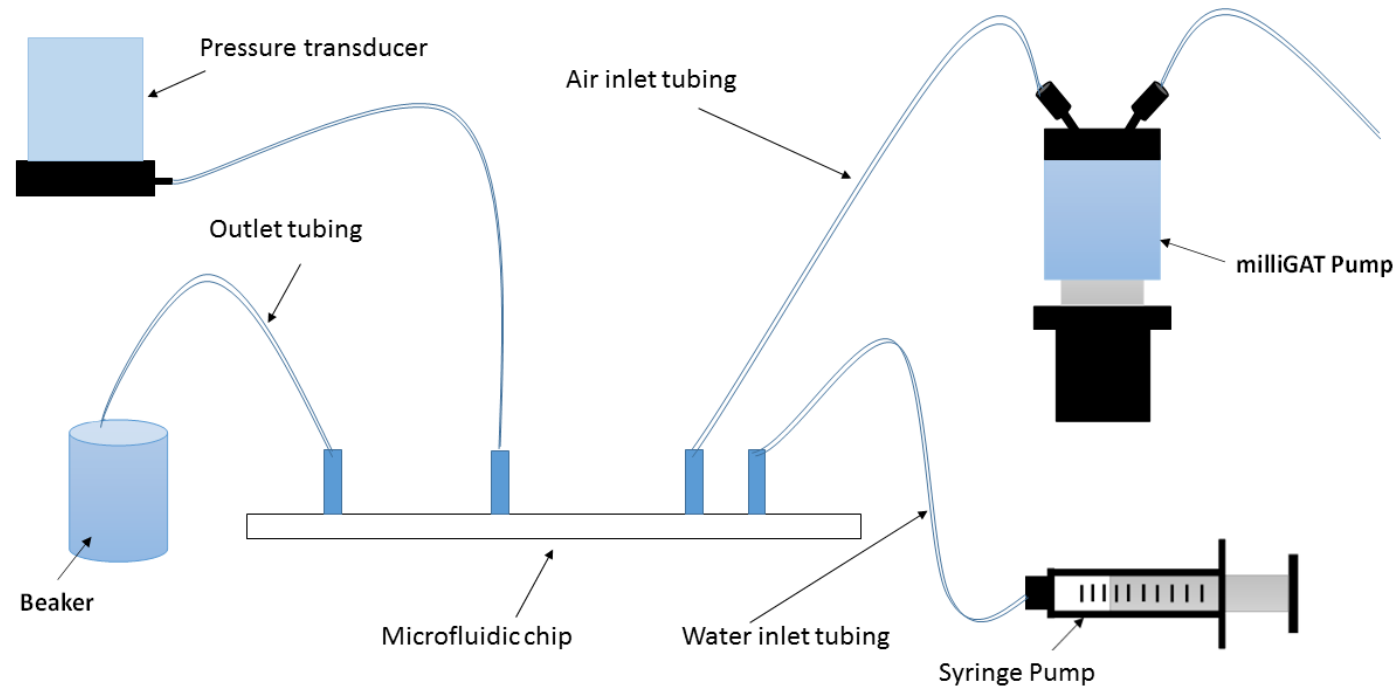

Figure 1 A schematic of the experimental setup

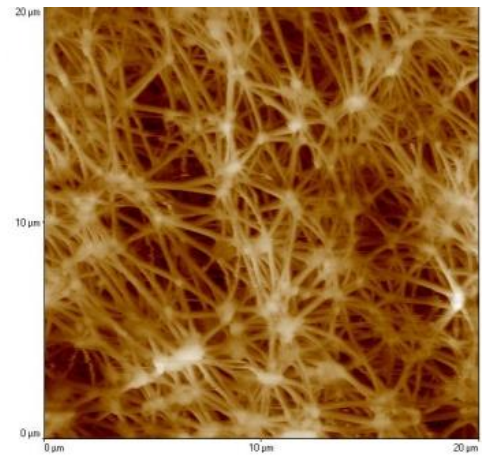

a

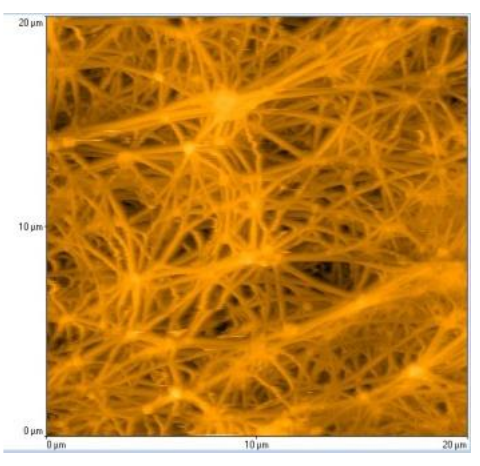

$\mathrm{c}$

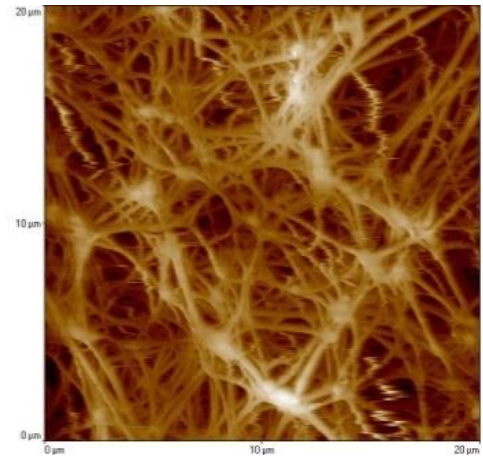

b

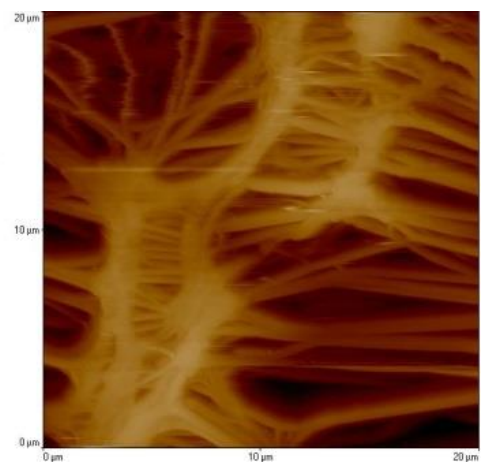

d

Figure 2 AFM images of various hydrophobic membranes used in this study. (a) $0.45-\mu \mathrm{m}$ Sterlitech membrane (b) $1-\mu \mathrm{m}$ Sterlitech membrane (c) 1- $\mu \mathrm{m}$ Pall membrane (d) 3- $\mu \mathrm{m}$ Advantec membrane 
Table 1 Specifications of the membranes used in this study*

\begin{tabular}{|c|c|c|c|c|}
\hline Pore Diameter & $0.45 \mu \mathrm{m}$ & $1.0 \mu \mathrm{m}$ & $1.0 \mu \mathrm{m}$ & $3.0 \mu \mathrm{m}$ \\
\hline Supplier & Sterlitech Corp. & Sterlitech Corp. & Pall Corp. & $\begin{array}{l}\text { Advantac mfs, } \\
\text { Inc. }\end{array}$ \\
\hline Porosity (\%) & 85 & 85 & NA & 83 \\
\hline Support material & $\begin{array}{l}\text { non-woven } \\
\text { polypropylene }\end{array}$ & $\begin{array}{l}\text { non-woven } \\
\text { polypropylene }\end{array}$ & $\begin{array}{l}\text { Spun bonded } \\
\text { non-woven } \\
\text { polyester }\end{array}$ & unsupported \\
\hline Thickness $(\mu \mathrm{m})$ & 175 & 175 & 216 & 75 \\
\hline Gurley Number & $4-10.5$ & $<6$ & 5.6 & NA \\
\hline $\begin{array}{l}\text { Water Breakthrough } \\
\text { Pressure }(\mathrm{kPa})\end{array}$ & 62.0 to 103.4 & 41.4 to 82.7 & 151.7 & $\geq 13.1$ \\
\hline Operating temperature & $130^{\circ} \mathrm{C}$ & $130^{\circ} \mathrm{C}$ & $250^{\circ} \mathrm{C}$ & $260^{\circ} \mathrm{C}$ \\
\hline
\end{tabular}

*Data provided by membrane suppliers

\subsection{Design and fabrication of the microfluidic chip}

A schematic of the microfluidic device is shown in Figure 3. The device consists of a glass substrate with inlet and outlet ports, fluidic channels, a PTFE membrane, and a base cover. The fluidic channels were designed in AutoCAD software and cut in a polyimide film using a programmable Craft Robo plotter (Graphtec Corporation, TX, USA). The fluidic channels that used in experiments were $500 \mu \mathrm{m}$ wide and $100 \mu \mathrm{m}, 200 \mu \mathrm{m}$, and $300 \mu \mathrm{m}$ deep. Four holes were drilled in the glass substrate using a micro milling machine for inlet, outlet, and pressure measurement ports. Plastic tubing was attached to the ports using an epoxy adhesive. A $1 \mathrm{~mm} \times 1$ $\mathrm{mm}$ opening was cut out in the base cover for bubble removal. A piece of desired membrane was cut and attached to the fluidic channel. In order to prevent leakage and bonding issues, membranes were cut sufficiently larger than the fluidic channel. Assembly was completed by aligning and bonding the glass plate, fluidic channels, and base cover. The device was cured by applying pressure and heating in a programmable oven at $80^{\circ} \mathrm{C}$ for 2 hours. The overall dimensions of the microfluidic device after assembly were approximately $25 \mathrm{~mm} \times 75 \mathrm{~mm} \times 2 \mathrm{~mm}$. 
Upper glass Cover With Tubing

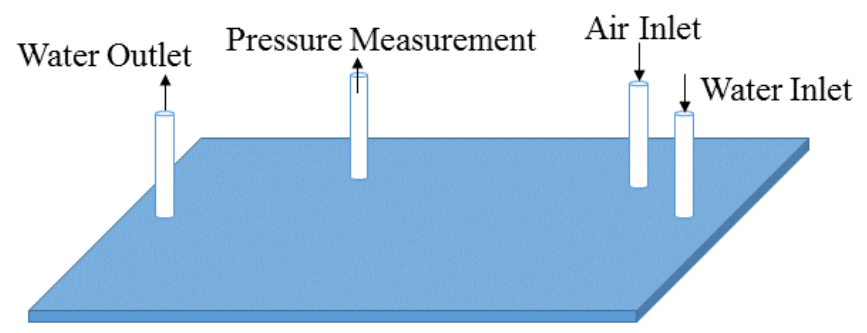

Fluidic Channel With Cavity

(Double Sided Tape)

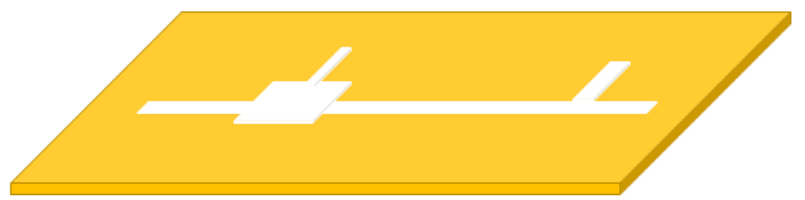

Hydrophobic Membrane

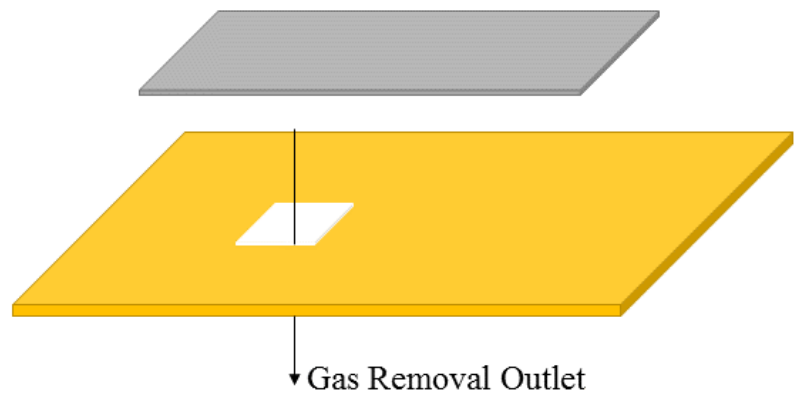

Lower Cover With Cavity Hole

(Double Sided Tape)

Figure 3 Fabrication and assembly of a microfluidic device for bubble removal

\subsection{Experimental procedure}

After fabrication and assembly of the microfluidic chip, experiments were conducted to determine the bubble removal capability of the device. Each test began by making fluidic connections between the microfluidic chip and the air and water pumps. The device was then tested for leaks and obstructions by pumping water into the device using a syringe pump. If no leak was detected, air was introduced into a T-junction using a bidirectional milliGAT pump (Global FIA, Inc. WA). Air and water were set to desired flow rates. Once a gas flow was established in the inlet port, the shear force from the wall and the surface tension force, pinched off the gas phase in the T-junction and formed periodic bubble-water slugs. The bubbles generated in the T-junction flowed towards a bubble removal region where a positive pressure forced out the air bubble through the membrane while water flowed along the fluidic channels to the outlet port and collected in a beaker. A high speed camera attached to an optical microscope was used to observe and record bubble formation and removal through the membrane. The captured images were later 
analyzed using ScopeImage 9.0 software to determine the membrane characteristics for complete bubble removal. For each membrane, the water was maintained at a desired flow rate while the air flow rate was increased in small increments. The outlet port was continuously monitored for the presence of air bubbles. If there were no air bubbles present in the outlet channel, the air flow rate was further increased until air bubbles were observed in the outlet. To obtain the maximum gas bubble removal rate, the experiment was repeated at the same water flow rate and by taking the average air flow rate between the complete and partial removal cases. If air bubbles were not observed at the outlet, the air flow rate was recorded as the maximum flow rate for complete removal. However, if bubbles were observed at the outlet, the last reading for complete removal was recorded as the maximum air flow rate for complete removal. This averaging process was estimated to introduce an error of up to $5 \%$ in some cases. Then, water flow rate was increased to the next level and the above procedures were repeated.

\section{Computational model and numerical approach}

\subsection{Model geometry and boundary conditions}

A schematic of the model geometry is shown in Fig. 4. Before performing a vast simulation study, a series of simulations were carried out with the same geometry and test conditions used in our experiments to validate our computational approach and methodology. However, to reduce the computational time, most of the simulations were performed using a smaller channel and cavity size than those used in the experiments. Unless stated otherwise, the simulations were performed for a microfluidic device with a channel width (w) of $100 \mu \mathrm{m}$ width, a channel depth (h) of 100 $\mu \mathrm{m}$, and a cavity size of $300 \mu \mathrm{m} \times 300 \mu \mathrm{m}$. At the inlets, fluid velocities were set for air and water phases. A zero gauge pressure was set at the outer surface of the porous membrane and a proper gauge pressure was applied at the channel outlet to compensate for the pressure drop caused by the outlet tubing. A no-slip boundary condition was applied at the wall. A contact angle of $72^{\circ}$ and $110^{\circ}$ (measured experimentally), were applied at the solid walls and hydrophobic membrane, respectively. Tetrahedron mesh was used in the fluidic channel section and cubic cells in the porous membrane region. To capture the air-water interface with a better contrast, the mesh size was refined in regions where sharper changes were likely to occur such as the T-junction and interfaces. 


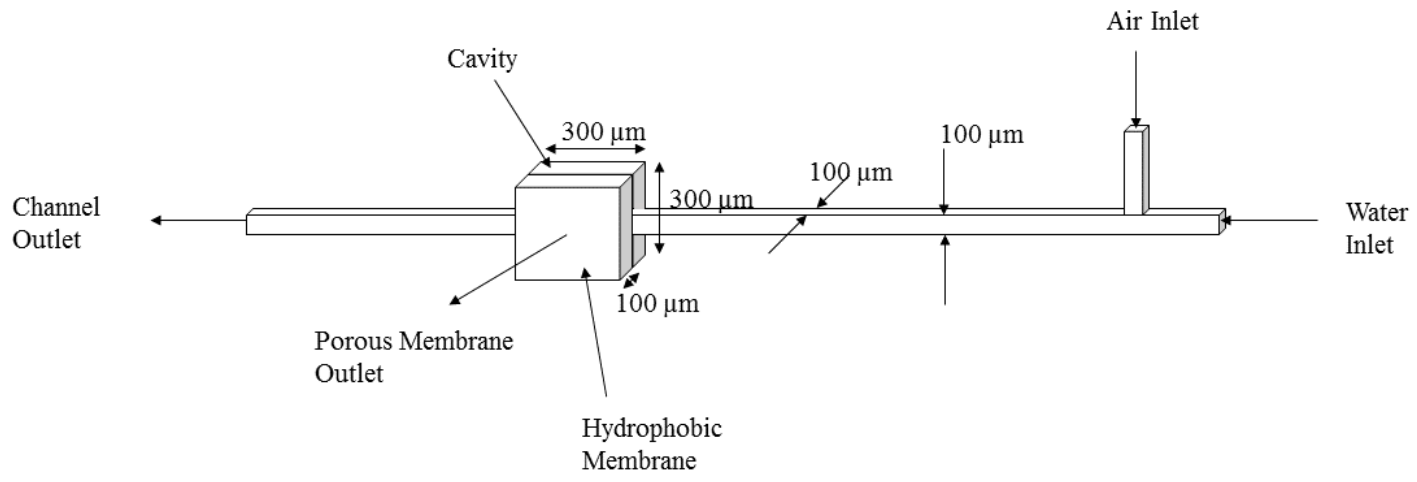

Figure 4 Model geometry used in the computational study

For the porous region in the computational model, the properties of the $1-\mu \mathrm{m}$ pore size membrane (Pall Corporation) were used. To obtain the permeability of the membrane, a series of tests at various gas flow rates were performed and the Darcy's equation was used to calculate the permeability value. In addition, the experimentally determined permeability was compared with several empirical or analytical permeability models available in the literature. Davies [36] derived an empirical correlation for layered fibrous structures as follows:

$\frac{k}{r^{2}}=\left[16 \emptyset^{1.5}\left(1+56 \emptyset^{3}\right)\right]^{-1}$

where $\varnothing$ is the solid volume fraction, $r$ is the radius of the fiber and $k$ is the permeability. Tomadakis et al. [37] used electrical conductivity and proposed an analytical equation for the permeability of the fibrous material with layered structures which is valid for the solid volume fractions of less than 0.3:

$\frac{k}{r^{2}}=\frac{\varepsilon}{8 \ln ^{2}(\varepsilon)} \frac{\left(\varepsilon-\varepsilon_{p}\right)^{\alpha+2}}{\left(1-\varepsilon_{p}\right)^{\alpha}\left[(\alpha+1) \varepsilon-\varepsilon_{p}\right]^{2}}$

where, $\varepsilon$ is the porosity of the porous media, $\alpha$ is a constant $(0.785)$ and $\varepsilon_{p}$ is the percolation threshold (0.11). Most of the analytical permeability equations are based on 2-D cell models. In these models, it is assumed that all fibers experience the same flow regime and the fibers are either 
parallel or perpendicular to the flow direction. An example of such models is an equation derived by Drummond et al. [38]:

$\frac{k}{r^{2}}=\frac{1}{8 \emptyset}\left(-\ln (\varnothing)-1.476+2 \varnothing-1.744 \emptyset^{2}\right)$

A comparison between these models and our experimentally determined permeability is shown in Table 2.

Table 2 Model predictions for permeability

\section{Model}

Davies (empirical)

Tomadakis (analytical)

Drummond (analytical)

Experimental
Permeability, $k\left(m^{2}\right)$

$\begin{array}{lc}9.05 \times 10^{-13} & +13.1 \\ 9.62 \times 10^{-13} & +20.1 \\ 5.68 \times 10^{-13} & -29.1 \\ 8.01 \times 10^{-13} & 0.0\end{array}$

\subsection{Governing Equations}

The computational domain consists of two regions, a microfluidic channel and a porous media section. For the fluidic channel section, we deal with a two-phase water-air Taylor flow. The governing equations for this region are as follows:

Conservation of mass:

$\frac{\partial}{\partial t}(\rho)+\nabla \cdot(\rho \vec{v})=0$

Conservation of momentum:

$\frac{\partial}{\partial t}(\rho \vec{v})+\nabla \cdot(\rho \vec{v} \vec{v})=-\nabla p+\nabla \cdot\left[\mu\left(\nabla \vec{v}+\nabla \vec{v}^{T}\right)\right]+\rho \vec{g}+\vec{F}$

To track the interface between the gas and liquid phases, VOF method [39] is utilized, which solves an extra equation for the gas phase volume fraction to distinguish the gas and liquid phases. The liquid phase volume fraction is computed using the gas phase volume fraction without the need to solve the VOF equation again. Assuming that there is no phase change, the volume fraction equation for the gas phase can be written as: 
$\left[\frac{\partial}{\partial t}\left(\alpha_{G} \rho_{G}\right)+\nabla \cdot\left(\alpha_{G} \rho_{G} \vec{v}\right)\right]=0$

$\alpha_{G}+\alpha_{L}=1$

where:

$\rho=\alpha_{G} \rho_{G}+\left(1-\alpha_{G}\right) \rho_{L}$

$\mu=\alpha_{G} \mu_{G}+\left(1-\alpha_{G}\right) \mu_{L}$

where $\alpha_{G}$ and $\alpha_{L}$ denote the volume fraction of gas and liquid phases, and $\mu_{G}$ and $\mu_{L}$ are the viscosity of gas and liquid phases, respectively. Term $\vec{F}$ accounts for the forces generated within the flow or exerted on the flow such as shear forces. The surface tension equation proposed by Brackbill et al. [40] is added to the momentum equation as a source term. The surface tension force can be expressed as a volume force using the divergence theorem:

$F=\sigma \frac{\rho K_{G} \nabla \alpha_{G}}{\frac{1}{2}\left(\rho_{G}+\rho_{L}\right)}$

where $\mathrm{K}$ is the curvature, defined as:

$K=\nabla \cdot \hat{n}$

$\hat{n}=\frac{n}{|n|}$

where ' $n$ ' is the surface normal, defined as:

$n=\nabla \alpha_{G}$

For the porous region, only the gas phase flows through the porous membrane. For such a small length scale and small fluid velocities $(\mathrm{v}<<1 \mathrm{~m} / \mathrm{s})$, the inertial terms can be neglected and the classical Darcy's law is valid [41]. The Darcy’s equation is given by:

$V=\frac{k}{\mu} \frac{\Delta p}{\Delta x}$

where $\mathrm{V}$ is the superficial velocity, $k$ is the permeability of the medium, and $\mu$ is the fluid viscosity.

\subsection{Numerical approach}


This is an author-created, un-copyedited version of an article published in Microsystem Technologies. The final publication is available online at DOI 10.1007/s00542-016-3020-2

A built-in VOF method in ANSYS FLUENT CFD software (Release 15.0) was utilized to track the air-water interface inside the fluidic channel. As reported by Taha et al. [32] and Qian et al. [5], the VOF model in FLUENT predicts the Taylor flow behavior with acceptable degree of accuracy. FLUENT uses the finite volume method to discretize the transport equations. The geometric reconstruction scheme is used for interface reconstruction. To relate the pressure and velocity corrections, a pressure-velocity coupling scheme, PISO (pressure-implicit with splitting of operators) is used. For pressure interpolation, PRESTO (pressure staggering option) scheme, for computing the gradient, least squares cell based method, and for momentum equation, the second order upwind discretization are used. The simulation results are analyzed using FLUENT postprocessor, Microsoft EXCEL, and MATLAB. Furthermore, suitable values for the relaxation factors, under relaxation factors, and courant number were chosen to fulfill the convergence criteria for different parameters.

\section{Results and Discussion}

\subsection{Experimental results}

The performance of four different hydrophobic membranes with different pore sizes was assessed for complete bubble removal in a microfluidic device. All experiments were performed using a microfluidic device with a channel width of $500 \mu \mathrm{m}$ and a cavity size of $1 \mathrm{~mm} \times 1 \mathrm{~mm}$. According to free energy of surfaces found in Young's equation [18], formation of a solid-gas interface on the membrane decreases the system energy. This means that the chance of gas bubbles to be in direct contact with the membrane increases. A porous membrane in its simplest form can be considered as an array of small cylindrical holes. These small holes are initially filled with air. When the membrane is brought in contact with water, menisci are formed in these pores which according to Laplace-Young pressure balance equation, prevent the water phase from leaking out through the membrane. This is a characteristic of the membrane and is governed by its breakthrough pressure. For the regions where membrane is in direct contact with air, the gas phase will diffuse through these small holes and work as a venting mechanism. The steady-state gas mass flux depends on the average time that the gas phase is in contact with the membrane, gauge pressure of the gas phase within the cavity and geometrical parameters such as the thickness and permeability of the membrane $(\mathrm{k})$. By changing any of these parameters, the bubble removal rate will change. However, once a particular membrane is chosen, geometrical parameters are fixed and the system 
behavior cannot change. The bubble removal rate can be only increased by increasing the gas phase gauge pressure within the cavity or increasing the average time that the gas phase is in contact with the membrane. Increasing the pressure inside the cavity, will increase the pressure of adjacent liquid phase too, which in turn decreases the likelihood of complete removal. In most cases, the residence time of the air inside the cavity decreases as the pressure inside the cavity increases. Hence, to simplify the discussion, the residence time of air inside the cavity is used to explain the bubble removal behavior of different configurations in this work.

Figure 5 shows optical microscope images of the bubble removal region. Air and water are introduced into the channel at preset flow rates. At the T-junction, the shear stress will cause the gas phase to form bubble slugs in the straight channel. Once the air bubbles reach the bubble removal region, they are trapped in the cavity and removed through the membrane. In this particular case, the water flow rate was kept at a $100 \mu \mathrm{L} / \mathrm{min}$, while the air flow rate was increased in $100 \mu \mathrm{L} / \mathrm{min}$ increments ranging from $100 \mu \mathrm{L} / \mathrm{min}$ to $400 \mu \mathrm{L} / \mathrm{min}$. For each case, the image was taken at an instance where the bubble in the cavity reached its maximum size. These images were taken at different times from the start of the experiments, since the time needed for each gas bubble to reach its maximum size differed based on the inlet flow rates. The results show that for a constant water flow rate, the bubble size in the cavity increases with increasing the air flow rate. However, if the air flow rate is further increased, gas bubble dynamics inside the cavity changes and the momentum toward the channel outlet is greater than momentum toward the porous membrane, preventing a portion of the gas bubbles from venting out through the membrane.

Figure 6 depicts the bubble removal rate as a function of water flow rate for different membranes of varying pore sizes. Regardless of the membrane pore size, the results show that the bubble removal rate decreases with increasing the liquid flow rate. As the liquid flow rate increases, the residence time of bubbles in the cavity decreases. Thus, the bubbles do not have sufficient time to escape through the membrane. Additionally, the pressure in the channel increases with increasing the water flow rate. However, the pressure inside the gas bubbles is greater than the adjacent water phase slug. Basically, the pressure difference between the gas bubble and environment governs the venting process. The pressure downstream of the cavity doesn't change significantly as the inlet flow rates change. Contrary to the porous membrane, there is no barrier at the downstream of the 
cavity to stop the gas bubble from moving toward the downstream channel. Thus, an increase in the water flow rate changes the balance inside the cavity in favor of partial removal and decreases the bubble removal capacity of the system. As seen in Fig. 6, for various combinations of air and water flow rates, the rate of bubble extraction increases as the pore size increases from 0.45 to 3.0 $\mu \mathrm{m}$ because permeability of the membrane increases as pore size increases. Flow behavior inside the cavity is largely dictated by the inlet flow rates and does not significantly depend on the membrane pore size. Higher bubble removal rates can be obtained by increasing the membrane pore size. However, the upper limit of the membrane pore size for a particular application is determined by its liquid breakthrough pressure.

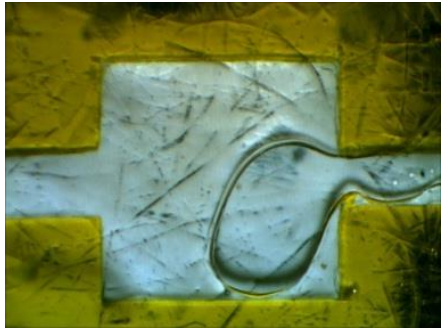

a

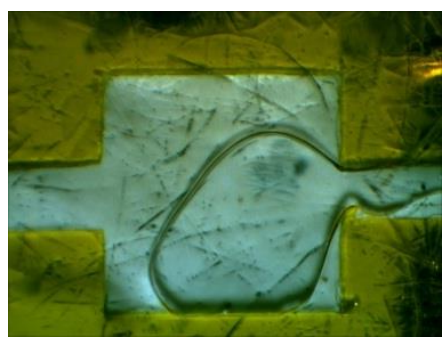

c

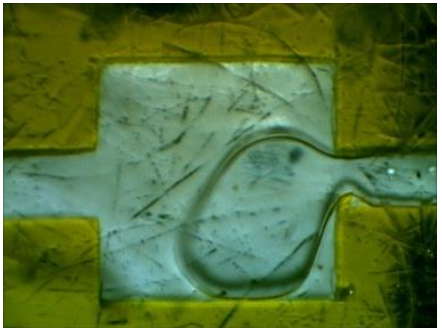

b

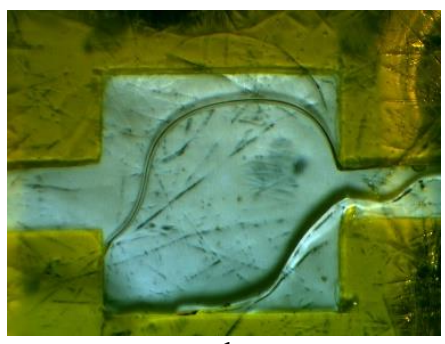

d

Figure 5 Microscope images of bubble removal region. The water flow rated was kept at $100 \mu \mathrm{L} / \mathrm{min}$ in all cases (a) air flow rate of $100 \mu \mathrm{L} / \mathrm{min}$; (b) air flow rate of $200 \mu \mathrm{L} / \mathrm{min}$; (c) air flow rate of $300 \mu \mathrm{L} / \mathrm{min}$; (d) air flow rate of $400 \mu \mathrm{L} / \mathrm{min}$. The channel depth is a $100 \mu \mathrm{m}$ in all cases. These images show the maximum gas bubble sizes at specified water and gas flow rates.

It is apparent from Fig. 6 that the bubble removal characteristics of the 1- $\mu$ m membranes from two different suppliers are quite different. Both of these membranes have a nominal pore size of $1 \mu \mathrm{m}$. However, the porosity and support material of these membranes are vastly different. As discussed previously, the $1-\mu \mathrm{m}$ Sterlitech membrane is laminated onto a polypropylene support material while the $1 \mu \mathrm{m}$ Pall membrane is spun bonded on non-woven polyester support with a much higher porosity. Microscope images of these support materials for these membranes are shown in Fig. 7. 
It can be seen from these images that the open area of the Sterlitech support material is less than $50 \%$. Thus, the effective porosity of the membrane/support is significantly lower than the membrane itself. As a result, the bubble removal rate of the $1 \mu \mathrm{m}$ Sterlitech membranes was found to be less than the Pall membrane with the same pore size. This demonstrates the effect of porosity in the permeability of the membranes which was discussed in computational model and numerical approach section.

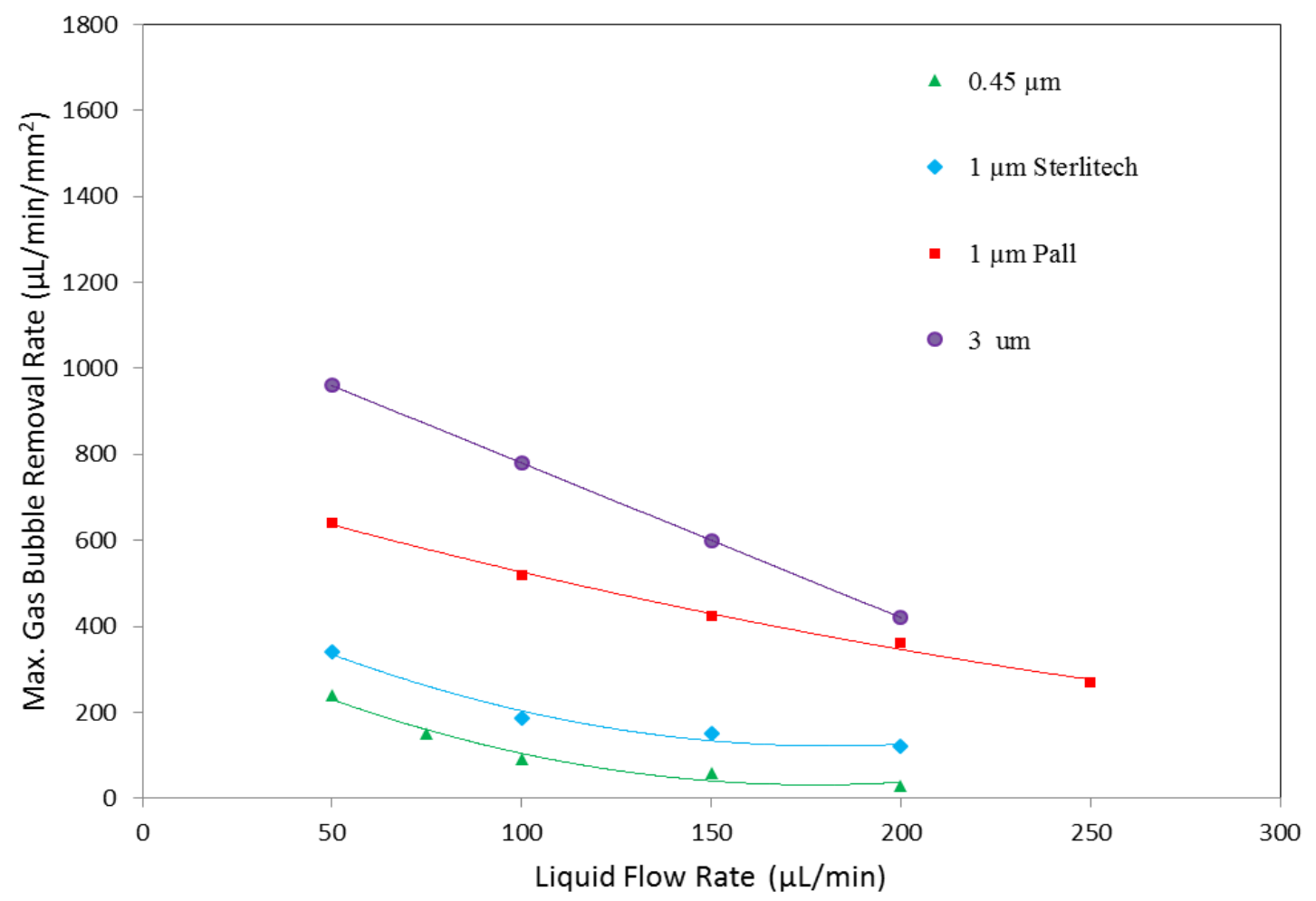

Figure 6 Maximum gas bubble removal rates per membrane area as a function of water flow rate for various membranes at a $100 \mu \mathrm{m}$ channel depth 


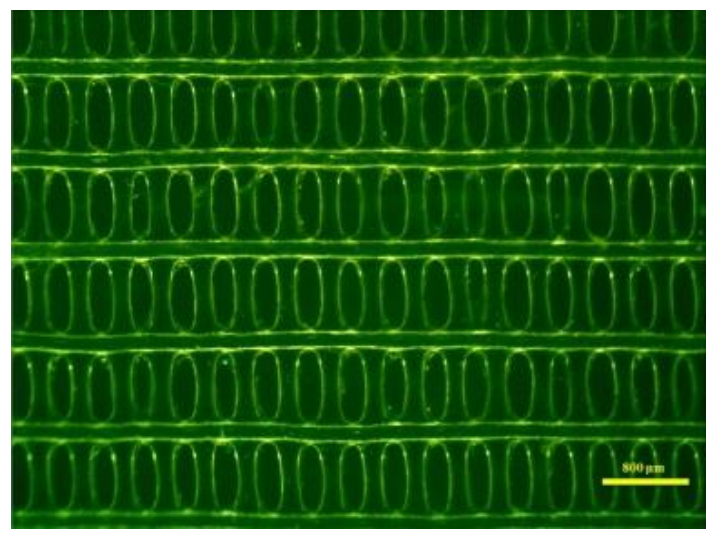

(a)

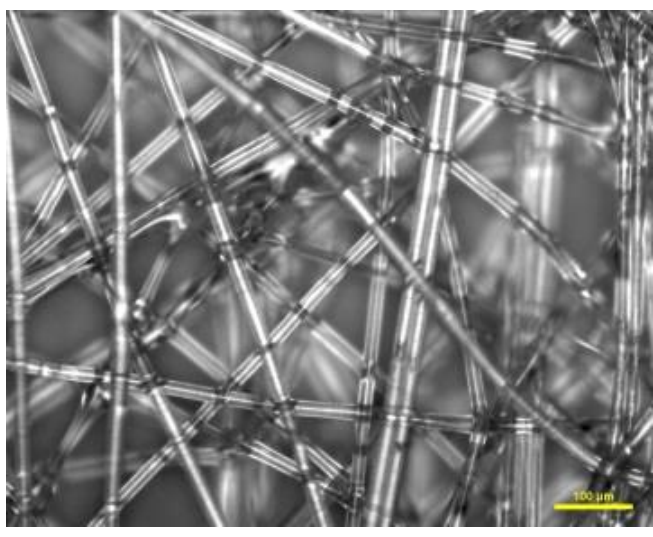

(b)

Figure 7 Optical Images of the support material for (a) $1 \mu \mathrm{m}$ - Sterlitech membrane; (b) $1 \mu \mathrm{m}$ - Pall membrane

The effect of channel depth on bubble removal rate is shown in Fig. 8. The results indicate that increasing the channel depth from $100 \mu \mathrm{m}$ to $300 \mu \mathrm{m}$ significantly increases the bubble removal rate per membrane surface area. This observation could be elucidated by the fact that for a given air flow rate, the bubble velocity decreases with increasing the channel depth. Thus, bubbles have a much longer residence time in the bubble removal region to escape through the membrane. On average, the bubble extraction rate for $200 \mu \mathrm{m}$ and $300 \mu \mathrm{m}$ channel depths are 2.7 and 3.3 fold higher than the $100 \mu \mathrm{m}$ channel depth, respectively. However, the increase is not linearly dependent on the channel depth and the rate of increase slows down as the channel depth increases. 


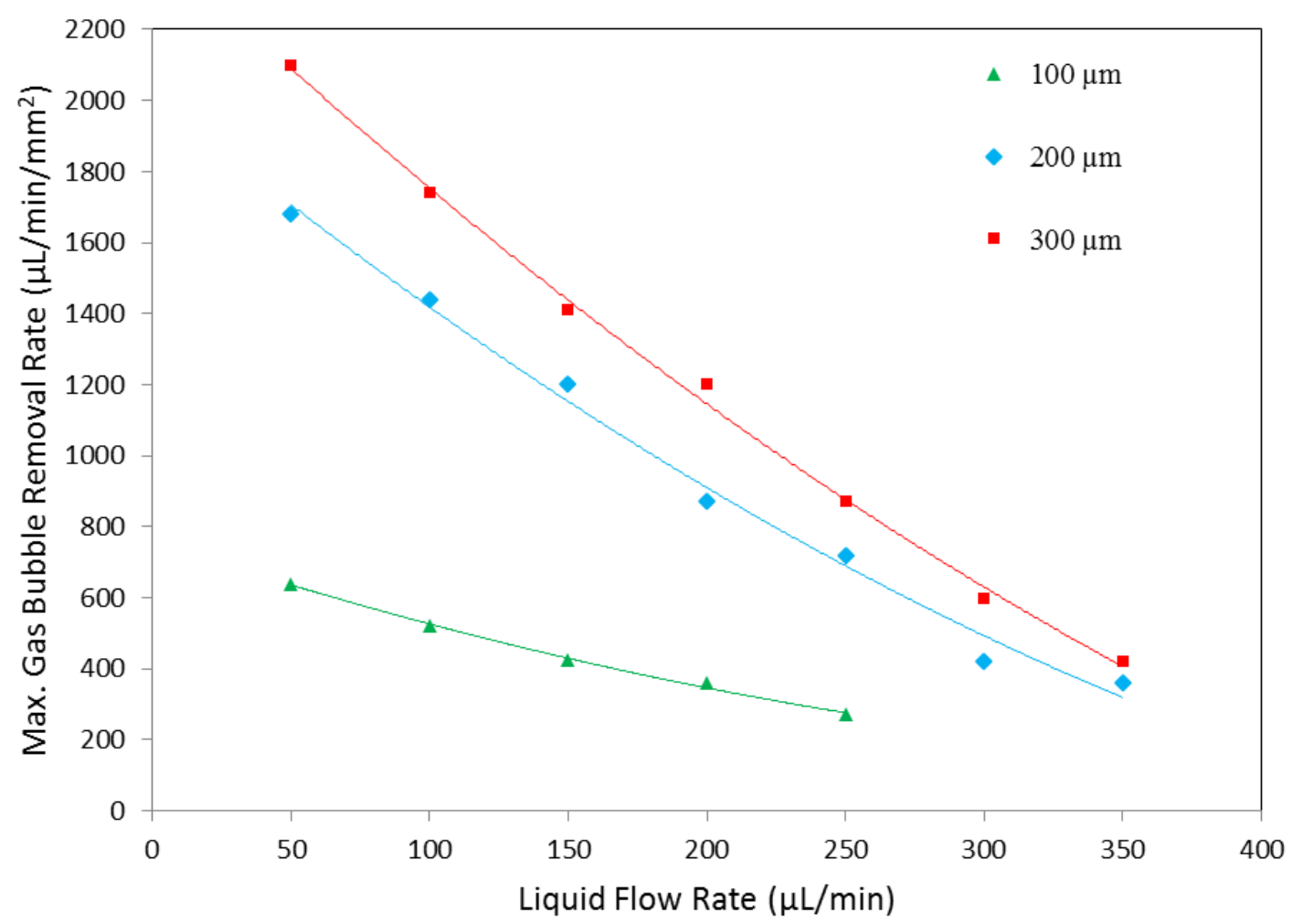

Figure 8 Bubble removal rate per membrane area for a $1.0 \mu \mathrm{m}$ Pall membrane at various channel depths

\subsection{Numerical results}

\subsubsection{Mesh independence analysis}

A series of simulations were performed with different number of elements in computational domain to ensure that the results were not mesh dependent. Figure 9 shows the volume fraction contours at different mesh resolutions. The results indicate that the interface is not captured correctly at low mesh resolutions and some defects are observed at the air-water interface. However, as the number of cells increases, a well-defined, sharp-interface is observed. Quantitatively, the effect of number of elements on gas bubble length was investigated to find the proper number of elements. Table 3 shows the changes in gas bubble length versus number of mesh elements. The results indicate that the percent error in bubble length decreases as the number of elements increases. When the percent error in gas bubble length is less than $1 \%$, the number of elements is considered to be adequate to obtain accurate results. Hence, 298721 elements was chosen to run the rest of simulations. Air is marked by red color and water by blue color in all of the images. For better clarity, 2D images of 3D simulations are presented here. 
(a)

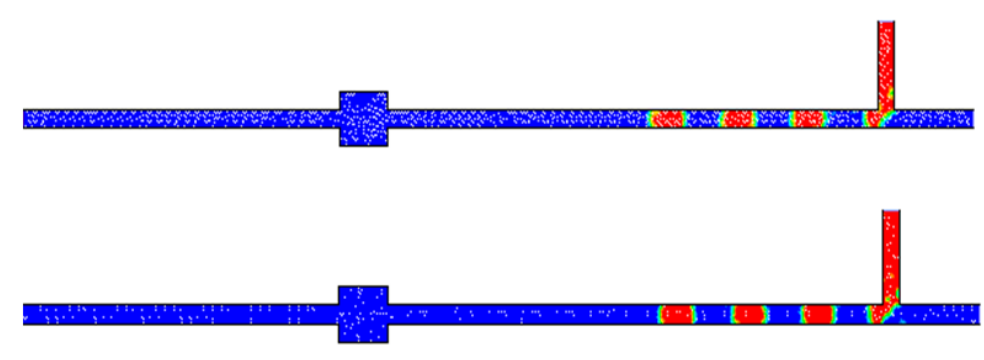

(b)

(c)

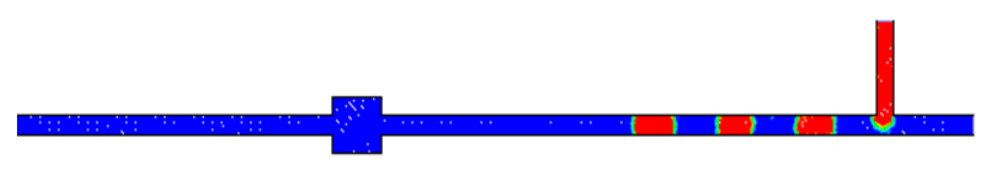

(d)

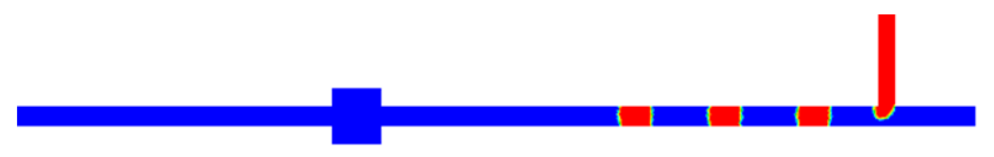

Figure 9 Volume fraction contours at various mesh resolutions for an air flow rate of $48 \mu \mathrm{L} / \mathrm{min}$ and a water flow rate of $60 \mu \mathrm{L} / \mathrm{min}$. The number of elements was (a) 68571 (b) 156971 (c) 212415 (d) 298721 , respectively. A well-defined interface is observed at higher mesh resolutions.

Table 3 Effect of number of elements on gas bubble length (channel width $=0.5 \mathrm{~mm}$, air and flow rate of $30 \mu \mathrm{L} / \mathrm{min})$

\begin{tabular}{lcc}
\hline \multicolumn{1}{c}{ Number of elements } & Gas bubble length $(\mathrm{mm})$ & Percentage change $(\%)$ \\
\hline 68571 & 1.506 & - \\
156971 & 1.569 & 4.18 \\
212415 & 1.614 & 2.86 \\
298721 & 1.640 & 1.61 \\
345324 & 1.651 & 0.67 \\
\hline
\end{tabular}

\subsubsection{Flow pattern and bubble removal characteristics}

Simulations were performed to study the flow pattern and bubble removal characteristics at various air/water inlet flow rates. The computational model used for the porous membrane is based on a single phase flow. Figure 10 presents sequential images of gas bubble/water slugs at various time steps along the channel. Initially, the channel is filled with water and the porous membrane is filled 
with air. The inlet stream is applied at time zero. After a while, air entering from the air inlet port meets the water phase at the T-junction where the air flow breaks into gas slugs inside the waterfilled channel and these gas slugs are transported through the channel until they reach the cavity. As shown in these figures, the air bubbles enter the cavity intermittently and are removed from the porous membrane normal to the flow direction. Since the porous membrane is hydrophobic, it repels the water molecules from its surface which leads to a higher contact area between the air and membrane surface as explained earlier. A higher contact area results in a higher gas removal rate. For these particular water and air inlet flow rates, the air bubbles have enough residence time in the cavity to be fully removed. The bubble removal capacity depends on the cavity dimensions, the porous membrane permeability, and the residence time of the bubble inside the cavity. The residence time of the gas bubbles depends on the water and gas flow rates and decreases when the air or water flow rate increases. Once a bubble enters the cavity and touches the membrane surface, it begins to permeate through the membrane. If the flow rate of the incoming gas bubbles is lower than the bubble removal capacity of the membrane, the bubble expands to a certain size and then shrinks as it vents through the membrane. With any new gas bubble entering the cavity, the bubble size alternately expands and shrinks again. The maximum size of the bubble within the cavity remains constant as long as the air and water flow rates do not change.

For a given cavity size and a membrane type, the maximum bubble removal capacity of the device could be determined based on flow behavior inside the cavity. Thus, the air and water flow rates have a major effect on the bubble removal rate. At a certain water flow rate, there exist a maximum air flow rate beyond which the system cannot fully remove the gas bubbles. Figure 11 depicts the time evolution for a partial gas bubble removal case at a water flow rate of $60 \mu \mathrm{L} / \mathrm{min}$ and air flow rate of $66 \mu \mathrm{L} / \mathrm{min}$. For this particular case, the gas bubbles cannot be fully removed by the membrane and they partially flow through the outlet channel for the reasons elaborated in the previous sections. 
This is an author-created, un-copyedited version of an article published in Microsystem Technologies. The final publication is available online at DOI 10.1007/s00542-016-3020-2

$16 \mathrm{~ms}$

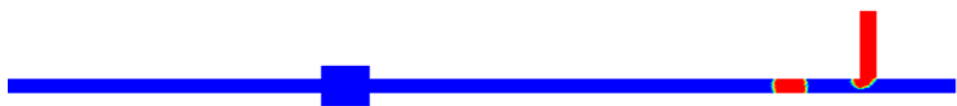

$36 \mathrm{~ms}$

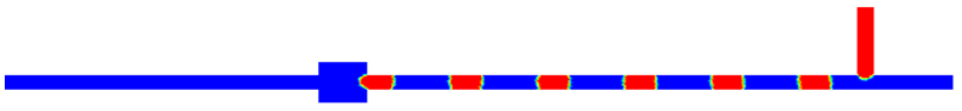

$42 \mathrm{~ms}$

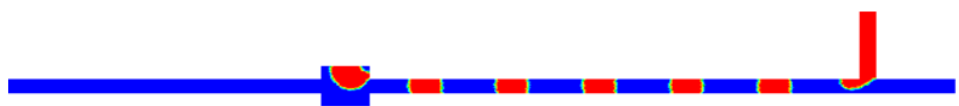

$45 \mathrm{~ms}$

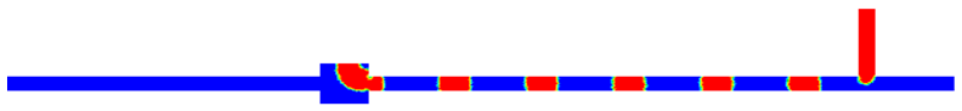

$50 \mathrm{~ms}$

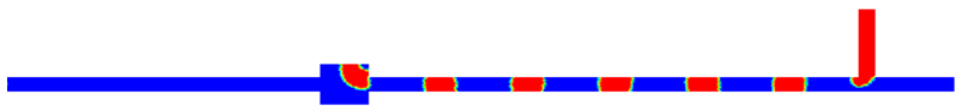

$51 \mathrm{~ms}$

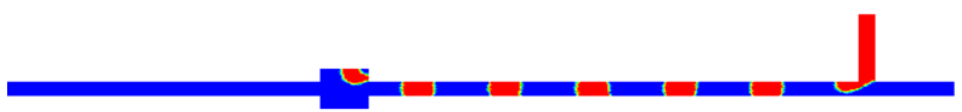

Figure 10 Time evolution of the gas bubble formation and removal at a water flow rate of $48 \mu \mathrm{L} / \mathrm{min}$ and an air flow rate of $30 \mu \mathrm{L} / \mathrm{min}$. The gas bubbles are fully removed in this case.

$20 \mathrm{~ms}$

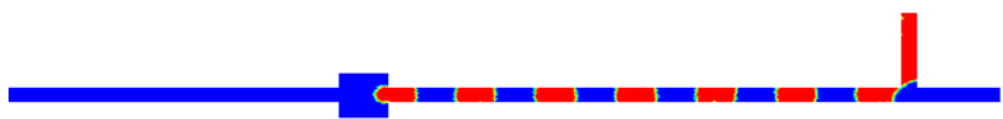

$22 \mathrm{~ms}$

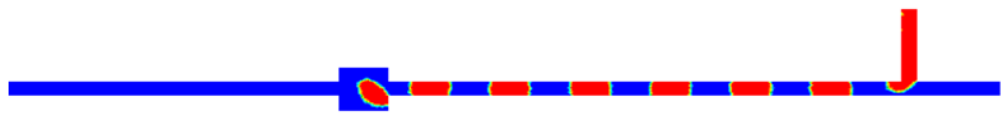

$23 \mathrm{~ms}$

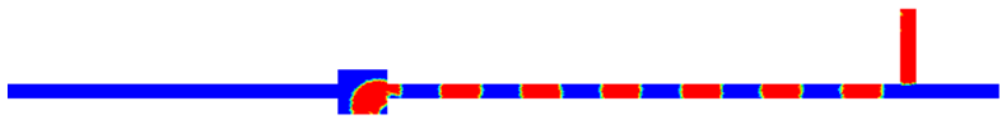

$25 \mathrm{~ms}$

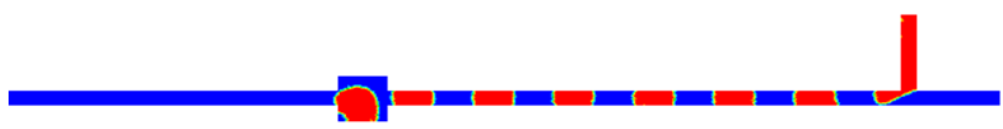

$26 \mathrm{~ms}$

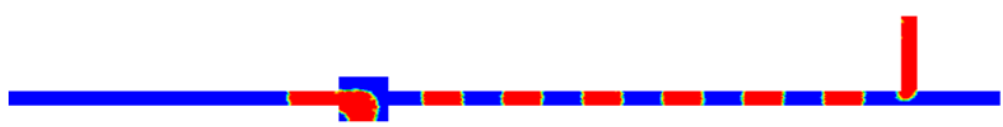

$29 \mathrm{~ms}$

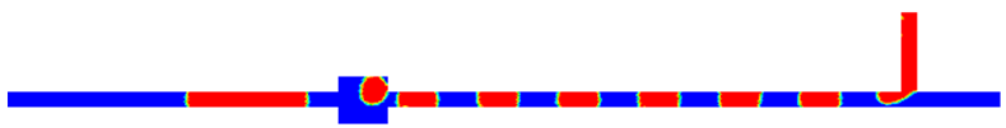

Figure 11 Time evolution of the gas bubble formation and removal at water flow rate of $60 \mu \mathrm{L} / \mathrm{min}$ and air flow rate of $66 \mu \mathrm{L} / \mathrm{min}$. The gas bubbles are partially removed in this case. 
Figure 12 depicts the flow pattern for partial bubble removal at a water flow rate of $60 \mu \mathrm{L} / \mathrm{min}$ and three different air flow rates of $36 \mu \mathrm{L} / \mathrm{min}, 48 \mu \mathrm{L} / \mathrm{min}$, and $54 \mu \mathrm{L} / \mathrm{min}$, respectively. As seen there, the frequency and size of the gas bubbles that flow through the outlet channel are different at different air flow rates. For this specific water flow rate, all of the bubbles entering the cavity are removed completely at an air flow of $30 \mu \mathrm{L} / \mathrm{min}$. At an air flow rate of $36 \mu \mathrm{L} / \mathrm{min}$, for every eleven bubbles that are removed, one bubble with a size of slightly larger than the incoming gas bubbles flow through the outlet channel. The bubble removal rate for this case is estimated to be approximately 88 percent of the incoming bubbles [42]. At an air flow rate of $48 \mu \mathrm{L} / \mathrm{min}$, for every five gas bubbles that enter the cavity, one gas bubble roughly 1.8 times the size of the incoming gas bubbles flows through the outlet channel, resulting in a bubble removal rate of 64 percent. For the air flow rate of $54 \mu \mathrm{L} / \mathrm{min}$, the gas removal rate is estimated to be 48 percent of the air inlet flow rate. At this air flow rate, for every four bubbles that enter the cavity, a larger bubble nearly twice the size of incoming bubbles flows through the outlet channel. For a given water flow rate, the percentage of partial removal decreases with increasing the air flow rate.

(a)

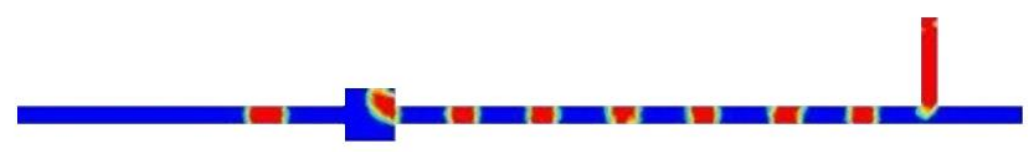

(b)

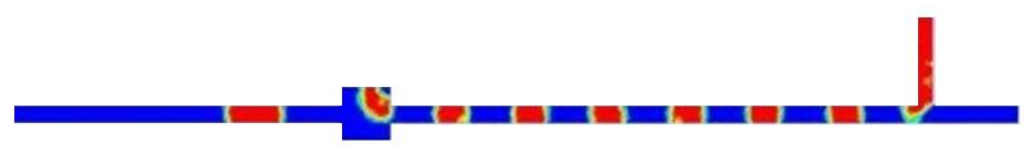

(c)

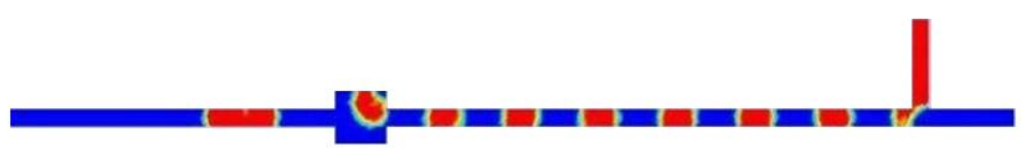

Figure 12 Partial bubble removal at water flow rate of $60 \mu \mathrm{L} / \mathrm{min}$ and air flow rates of (a) $36 \mu \mathrm{L} / \mathrm{min}$ (b) $48 \mu \mathrm{L} / \min$ (c) $54 \mu \mathrm{L} / \mathrm{min}$. The bubble removal rates for these cases are estimated to be approximately 88 , 64, and 48 percent, respectively. 


\subsubsection{Pressure distribution}

Figure 13 shows the contour plots of the volume fraction and the total pressure along the channel at a water flow rate of $60 \mu \mathrm{L} / \mathrm{min}$ and air flow rate of $48 \mu \mathrm{L} / \mathrm{min}$. The corresponding pressure distribution is shown in Fig. 14. The results indicate that the total pressure inside the air slugs is much higher than their adjacent water slugs. The peaks and valleys represent the pressure in the air capsules and water slugs, respectively. It is also observed that the pressure at the rear end of the gas bubbles is higher than their front end. Upstream of the bubble removal cavity, the pressure decreases along the channel. The total pressure increases inside the cavity since the cavity serves as an expansion device and the fluid velocity slows down within the cavity. The pressure then decreases along the channel downstream of the cavity.
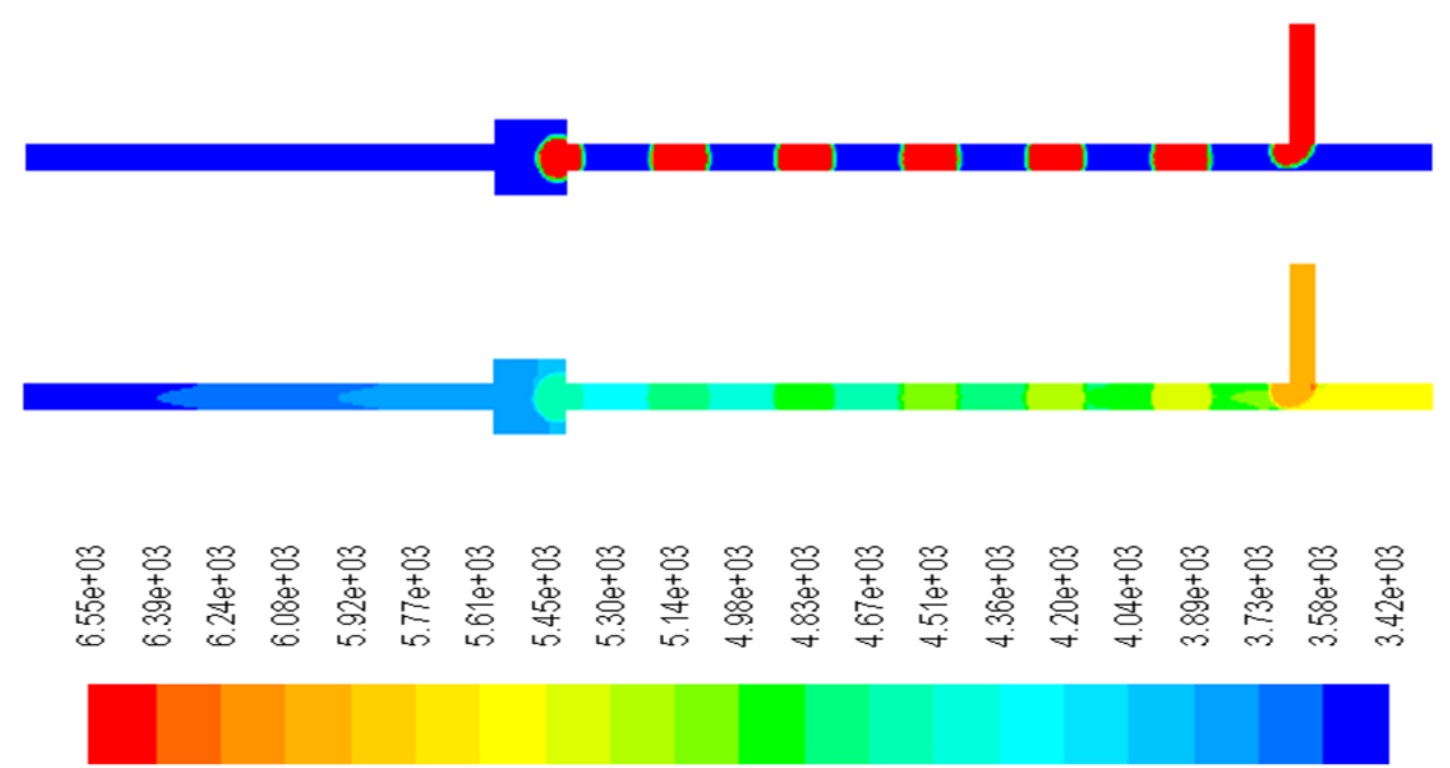

Figure 13 Contour plots of a) volume fraction and b) total pressure along the channel at a water flow rate of $60 \mu \mathrm{L} / \mathrm{min}$ and an air flow rate of $48 \mu \mathrm{L} / \mathrm{min}$. 


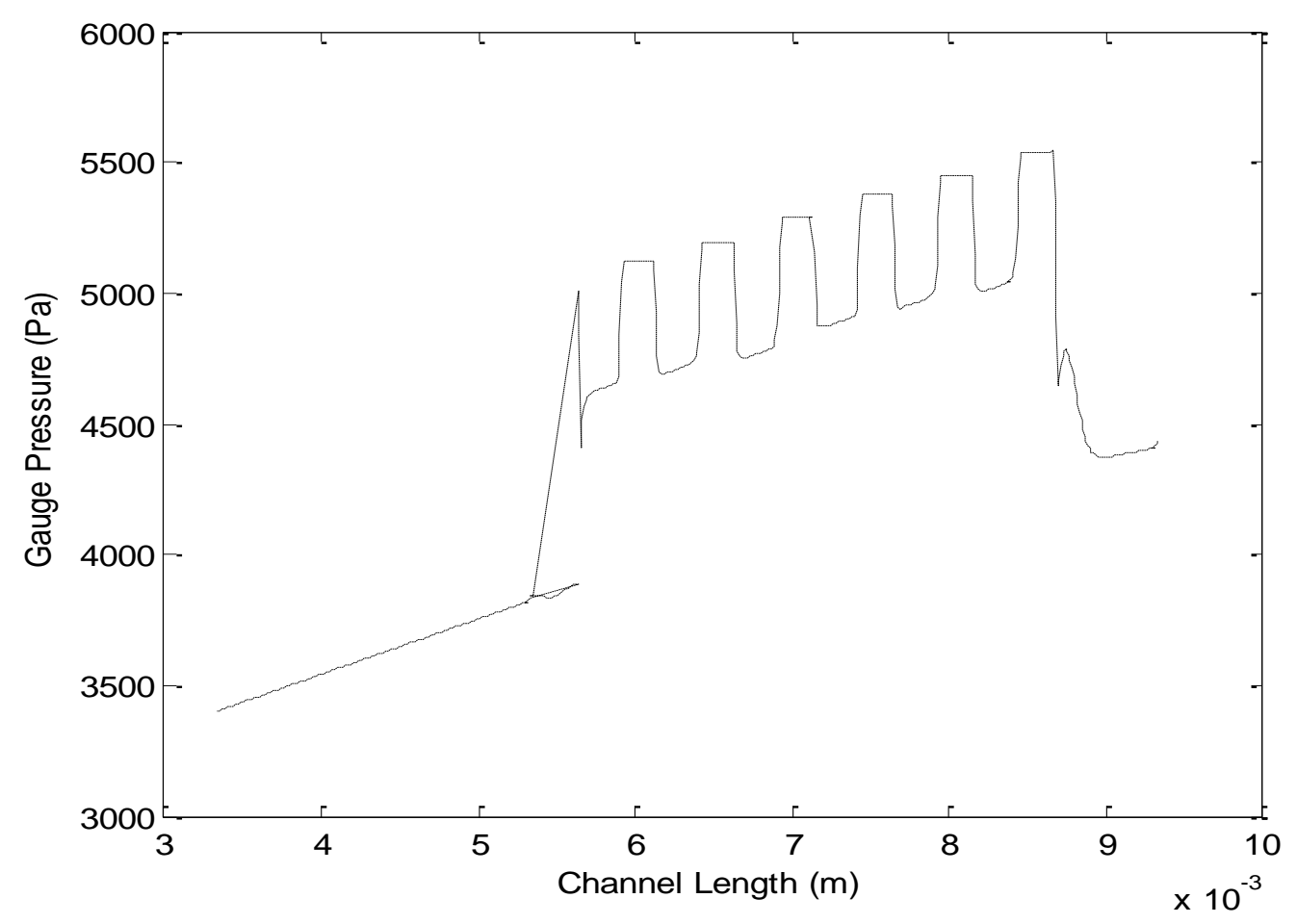

Figure 14 Pressure distribution along the channel at a water flow rate of $60 \mu \mathrm{L} / \mathrm{min}$ and an air flow rate of $48 \mu \mathrm{L} / \mathrm{min}$.

\subsubsection{Bubble extraction analysis}

To compare our computational and experimental results, we ran a series of simulations with the same geometry used in our experiments, which consisted of a channel width of $500 \mu \mathrm{m}$, a channel depth of $100 \mu \mathrm{m}$, a $1 \mathrm{~mm} \times 1 \mathrm{~mm}$ square cavity and a membrane pore size of $1.0 \mu \mathrm{m}$. Figure 15 shows a comparison between the experimental and simulation results. The results indicate a very good agreement between the simulations and experimental data. The difference is within $\pm 10 \%$. The results show that the bubble extraction capability of the system reduces with increasing the water inlet flow rate. This is because when the water flow rate increases, the residence time of the gas bubbles within the cavity decreases, resulting in a lower rate of air removal through the membrane. The bubble removal characteristics of the membrane depends on the contact area of the air bubbles with the membrane surface, pressure drop across the membrane, and the residence time of the air bubbles within the cavity. For a constant pressure drop across the membrane, higher contact area and residence time result in a higher rate of air removal. 


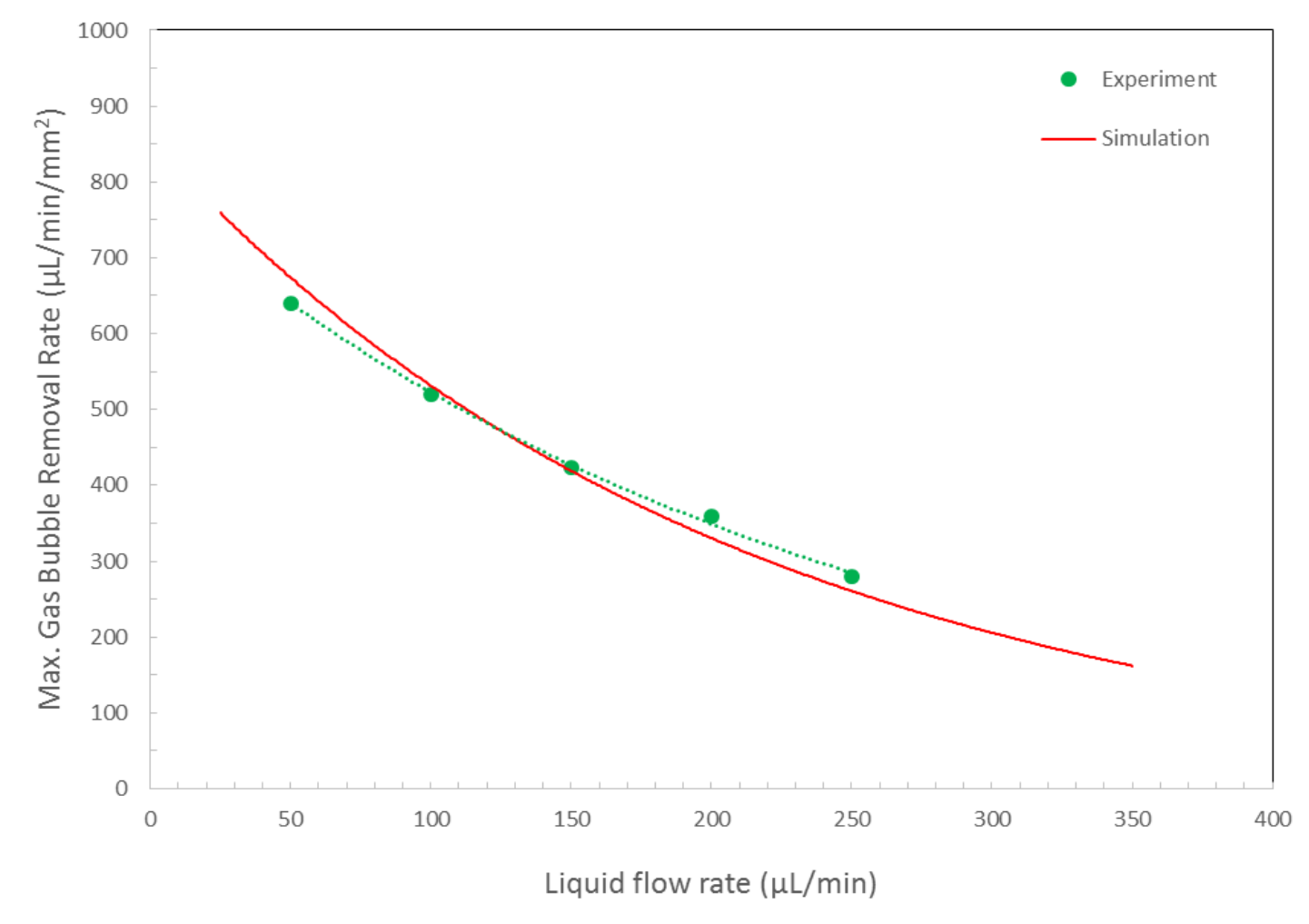

Figure 15 Comparison between the experimental data and simulation results of gas bubble removal per membrane area for a channel width of $500 \mu \mathrm{m}$, a channel depth $100 \mu \mathrm{m}$, a cavity size of $1 \mathrm{~mm} \times 1 \mathrm{~mm}$, and a membrane pore size of a $1.0 \mu \mathrm{m}$.

After validation of our numerical model, we used our model to investigate the effects of different parameters on the bubble removal capacity of the device. In order to further investigate the effect of geometry on bubble removal rate, two different channel widths of $100 \mu \mathrm{m}$ and $200 \mu \mathrm{m}$, with a $100 \mu \mathrm{m}$ channel depth, and an aspect ratio of 3 were simulated. The aspect ratio (AR) in this study is defined as the ratio of the cavity width to the channel width. Figure 16 shows the bubble extraction rate at different channel widths. The rate of bubble extraction per membrane area increases when the channel width increases from $100 \mu \mathrm{m}$ to $200 \mu \mathrm{m}$. 


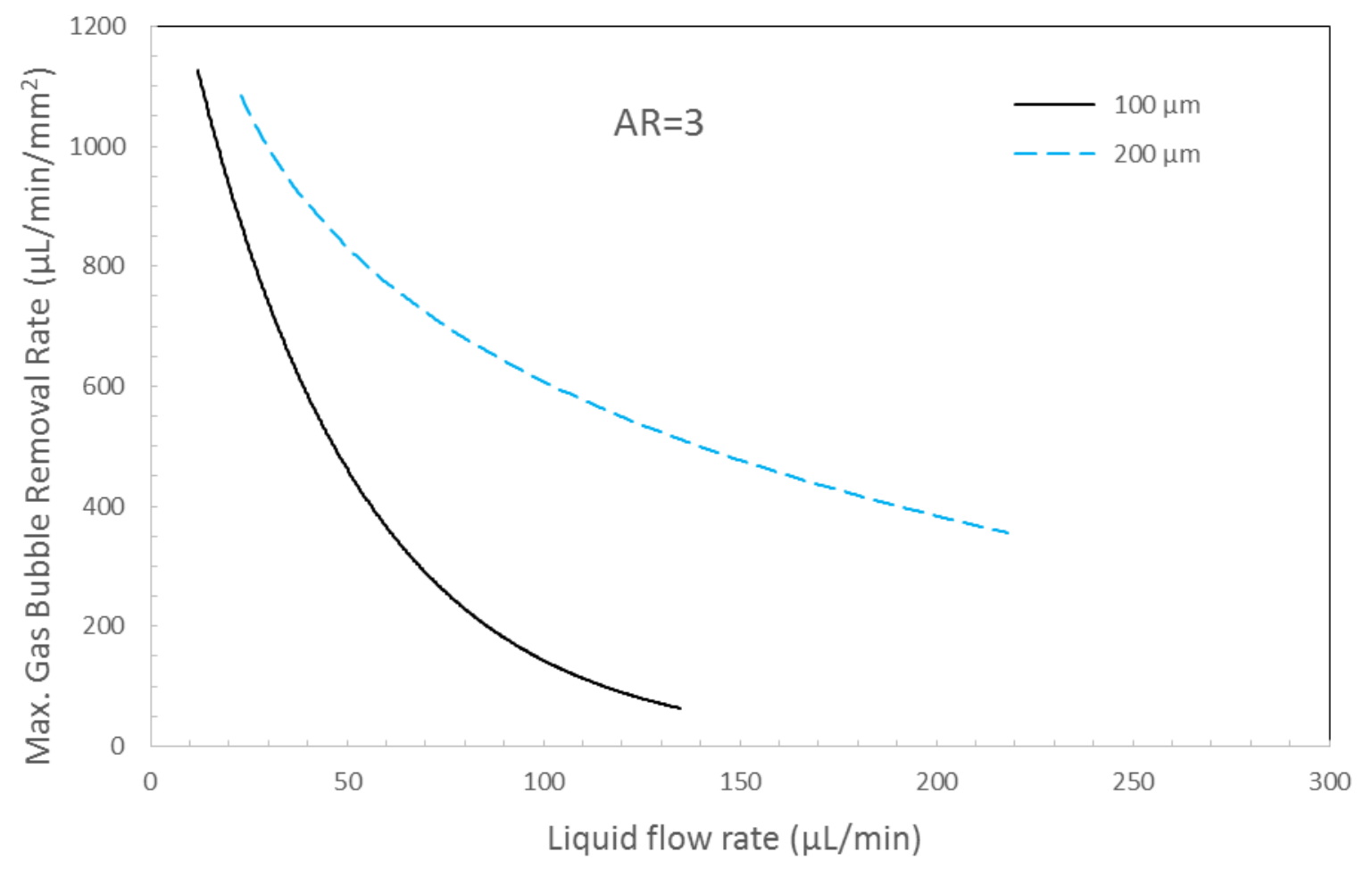

Figure 16 Effect of channel width on gas bubble removal. The depth and aspect ratio of the channels are $100 \mu \mathrm{m}$ and $\mathrm{AR}=3$, respectively.

The effect of cavity dimension on bubble extraction characteristics is shown in Fig. 17. The channel width and depth were fixed at $200 \mu \mathrm{m}$ and $100 \mu \mathrm{m}$, respectively, but the ratio of cavity width to channel width was changed from 3 to 2 . The results show that the rate of bubble extraction per membrane area increases when the aspect ratio decreases from 3 to 2 , indicating a better bubble removal efficiency. When the aspect ratio decreases both the cavity size and the amount of air removal decrease. However, the decrease in the cavity size is more dramatic than the amount of air removed which causes the normalized bubble removal rate per unit area of the membrane to increase. This observation can be explained by the fact that for $A R=2$, the percentage of the membrane area which is in contact with the gas phase at a particular water flow rate is higher than that of $\mathrm{AR}=3$. This means at $\mathrm{AR}=2$, a larger portion of the membrane is venting the gas bubbles out, which in turn increases the overall normalized bubble removal rate per membrane area of the device. 


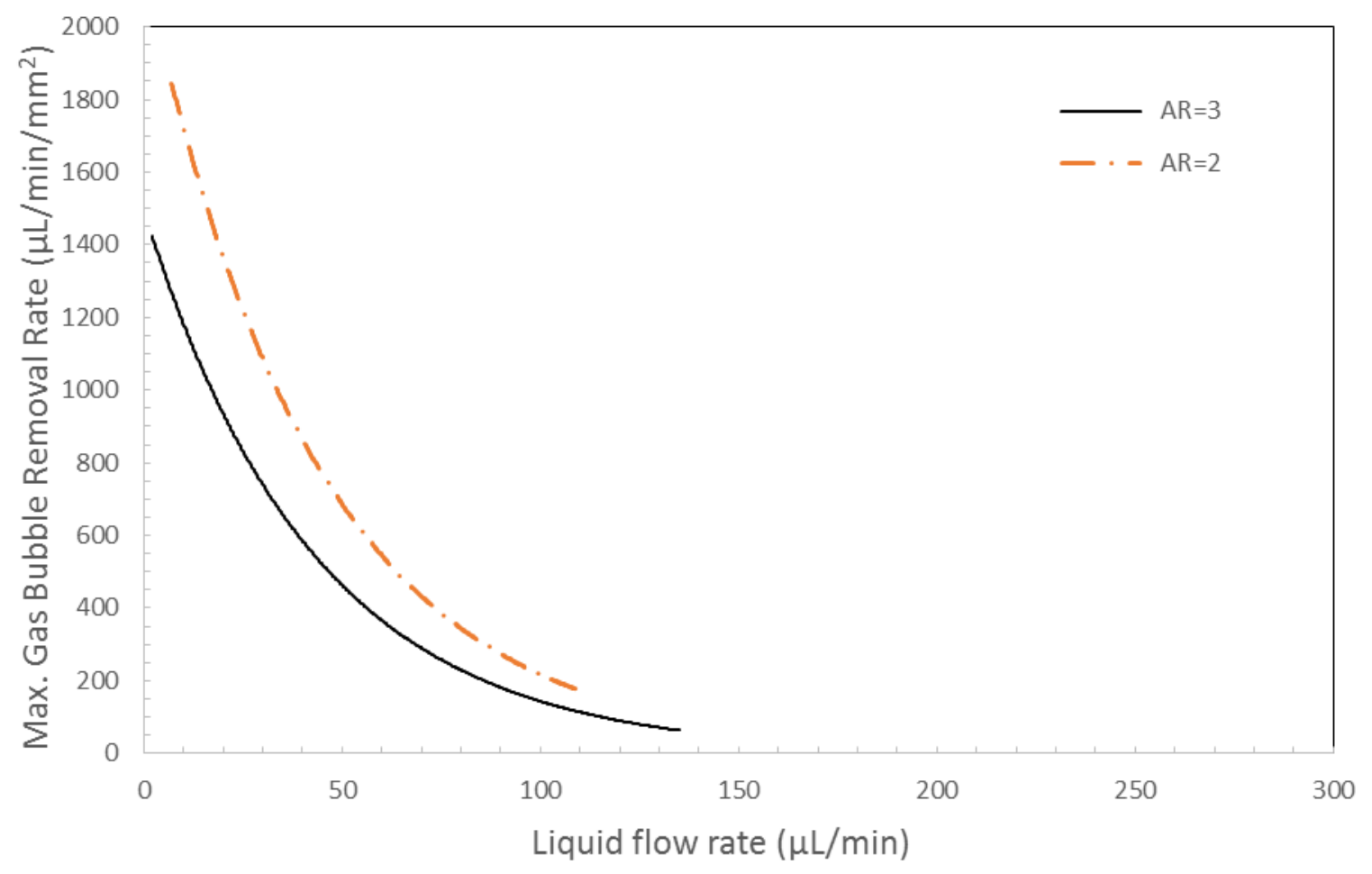

Figure 17 Effect of cavity size on bubble removal rate per membrane area at different aspect ratios. Channels' width and depth are $200 \mu \mathrm{m}$ and $100 \mu \mathrm{m}$, respectively.

Finally, simulations were also performed to study the effect of cavity inlet and outlet configuration on bubble extraction rate. A schematic of two different "in-line" and "off-set" configurations is shown in Fig. 18. All the results presented thus far were obtained for an in-line configuration. A comparison between these two configurations is presented in Fig. 19. The channel width and depth are $200 \mu \mathrm{m}$ and $100 \mu \mathrm{m}$, respectively, and the cavity size is $600 \mu \mathrm{m} \times 600 \mu \mathrm{m}$. The results indicate that the rate of bubble extraction per unit area for the offset configuration is significantly higher than the in-line configuration. This is attributed to a larger residence time of the bubbles within the cavity for the offset configuration and a larger contact area between the membrane and the gas phase. 

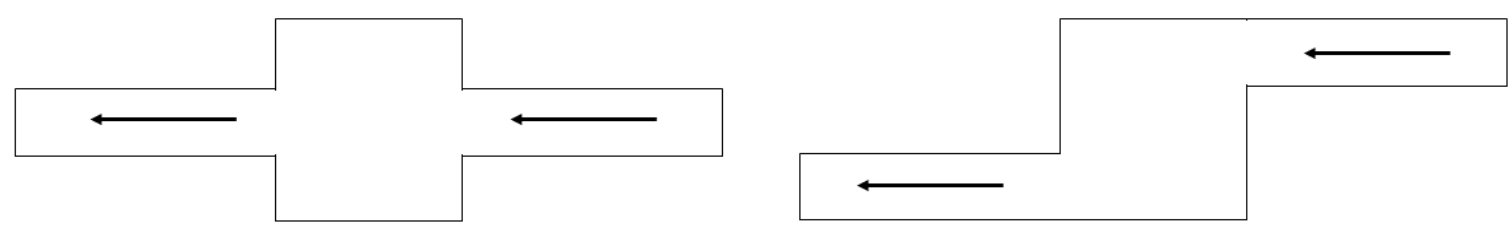

In-line

Offset

Figure 18 A schematic of different cavity inlet and outlet configurations.

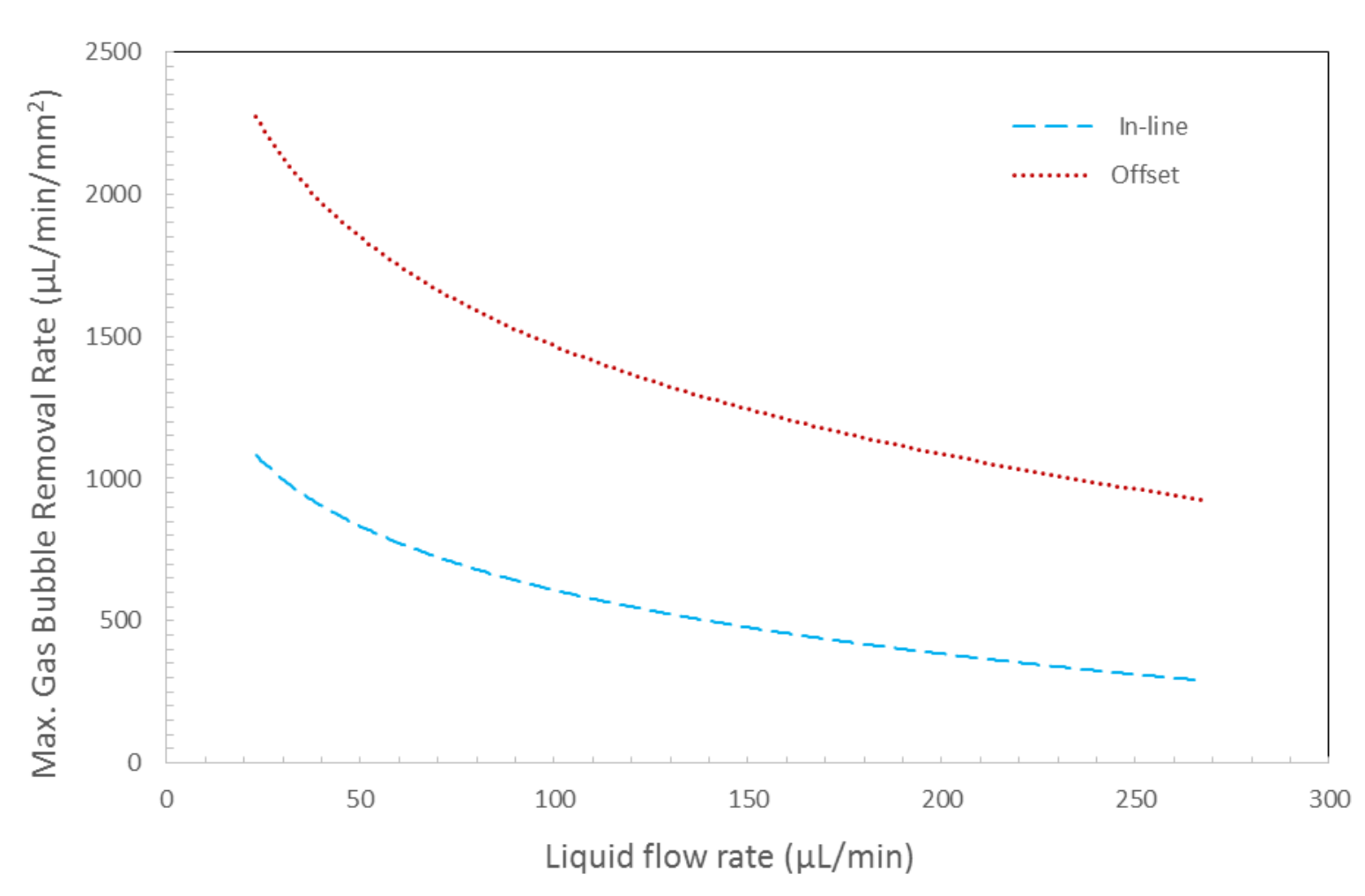

Figure 19 Effect of cavity inlet and outlet configurations on bubble removal rate. Channels' width and depth are $200 \mu \mathrm{m}$ and $100 \mu \mathrm{m}$, respectively.

\section{Conclusions}

In this study, a microfluidic device was deigned, fabricated and tested to determine bubble removal characteristics of several nanofibrous PTFE membranes. Additionally, a 3D computational model was developed to simulate bubble generation and removal, and to gain a better understanding of 
the phenomenon. The simulation results were compared with the experimental data both qualitatively and quantitatively. The agreement between computational results and experimental data was within $\pm 10 \%$. Once validated the model was used to study the effects of various geometric and operating parameters on the bubble extraction rate. The bubble extraction rate per membrane area was found to increase with increasing the channel depth and decrease with increasing the water flow rate. The gas phase size within the cavity was observed to alternately expand and shrink between its maximum and minimum volumes during the complete bubble removal process. For a given geometrical configuration, when the water flow rate was increased beyond a certain value, the gas bubbles could not be fully removed and partially passed through the outlet channel. The size and frequency of the bubbles in the outlet channel increased with increasing the water flow rate. The results of this study will help in selecting an appropriate configuration and membrane type for use in membrane-based bubble venting applications.

\section{Acknowledgments}

This work was partially supported by a RGGS grant from the SIUE Graduate School.

\section{References}

1. M. Mehling and S. Tay, 2014, Microfluidic cell culture, J of Current Opinion in Biotechnology Volume 25, pp $95-102$.

2. L. R. Damgaard, L. H. Larsen, N.P. Revsbech, 1995, Microscale biosensors for environmental monitoring, $\mathrm{J}$ of Trends in Analytical Chemistry, vol. 14. Issue 7, pp 300-303.

3. J.M. Karlsson, T. Haraldsson, S. Laakso, A. Virtanen, M. Maki, G. Ronan, W. van der Wijngaart, 2011, PCR on a PDMS-based microchip with integrated bubble removal, IEEE 2011 , pp $2215-2218$.

4. Y.S. Shin, K. Cho, S.H. Lim, S. Chung, S-J. Park, C. Chung, D-C. Han, J.K. Chang, 2003, PDMS-based micro PCR chip with Parylene coating, J Micromech Microeng, 13, pp. 76874.

5. D. Qian, A. Lawal, 2006, Numerical study on gas and liquid slugs for Taylor flow in a Tjunction microchannel, Chemical Engineering Science Volume 61, Issue 23, pp 76097625. 
6. M.T. Kreutzer, F. Kapteijn, J.A. Moulijn, C.R. Kleijn and J. J. Heiszwolf, 2005, Inertial and interfacial effects on pressure drop of Taylor flow in capillaries, AIChE Journal Volume 51, Issue 9, pp 2428-2440.

7. T. Taha, Z.F. Cui, 2004, Hydrodynamics of slug flow inside capillaries, Chemical Engineering Science, Volume 59, Issue 6, pp 1181-1190.

8. J.J. Heiszwolf, M.T. Kreutzer, M.G. van den Eijnden, F. Kapteijn, J.A. Moulijn, 2001, Gasliquid mass transfer of aqueous Taylor flow in monoliths, Catalysis Today Volume 69, Issues 1-4, pp 51-55.

9. J. Tan, S.W. Li, K. Wang, G.S. Luo., 2006, Gas-liquid flow in T-junction microfluidic devices with a new perpendicular rupturing flow route, Chemical Engineering Journal, 146, pp.428-433.

10. D. R. Link, S. L. Anna, D. A. Weitz, H. A. Stone., 2004, Geometrically Mediated Breakup of Drops in Microfluidic Devices, Physical Review Letters, 92, pp. 545031-34.

11. J. H. Xu, S. W. Li, G. G. Chen, and G. S. Luo., 2006, "Formation of monodisperse microbubbles in a microfluidic device", AIChE journal, 52, pp.2254 -2259.

12. P. Garstecki, M.J. Fuerstman, H.A. Stone, G.M. Whitesides, 2006, Formation of droplets and bubbles in a microfluidic T-junction-Scaling and mechanism of break-up, Lab on a Chip, 6, pp. 437-446.

13. T.T. Fu, Y. Ma, D. Funfschilling, H.Z. Li., 2009, Bubble formation and breakup mechanism in a microfluidic flow-focusing device, Chemical Engineering Science, 64, pp. 2392-2400.

14. R. Xiong, M. B. and J.N Chung, 2007, Formation of bubbles in a simple co-flowing microchannel, Journal of Micromechanics and Microengineering, 17, pp.1002-1011.

15. P. Garstecki, I. Gitlin, W. DiLuzio, G.M. Whitesides, E. Kumacheva and H.A. Stone., 2004, Formation of monodisperse bubbles in a microfluidic flow-focusing device, Applied Physics Letters, 85, pp.2649-2652.

16. T.T. Fu, X. Hui, C-Y Zhu, Y-G Ma, H-Z Li, 2011, Formation of dispersed small bubbles in flow-focusing microchannels, Journal of Chemical Engineering of Chinese Universities, 25, pp.337-340.

17. M.W. Grinstaff and K.S. Suslick, 1991, Air-filled proteinaceous microbubbles: synthesis of an echo-contrast agent, Proc. Natl. Acad. Sci., Vol. 88, pp 7708-7710. 
18. D.D. Meng, J. Kim and C-J Kim, 2006, A degassing plate with hydrophobic bubble capture and distributed venting for microfluidic devices, Journal of Micromechanics and Microengineering, Volume 16, pp 419-424.

19. JH Tsai and L. Lin, 2002, Active microfluidic mixer and gas bubble filter driven by thermal bubble micropump, J of Sens Actuators Vol. 97-98, pp 665-671.

20. A. Skelley and M.J. Voldman, 2008, An active bubble trap and debubbler for microfluidic systems, Lab Chip Vol. 8, 1733-1737.

21. Z. Yuan, Y. Zhang, Z. Li, Y. Zhao and X. Liu, 2014, Investigation of mass transport and cell performance on $\mu \mathrm{DMFC}$ with different anode flow fields, Int. J. Energy, 38, pp 139150.

22. C. Lochovsky, S. Yasotharan and A. Günther, 2012, Bubbles no more: in-plane trapping and removal of bubbles in microfluidic devices, Lab on a Chip, 12, Issue 3, pp 595-601.

23. M. Johnson, G. Liddiard, M. Eddings, and B. Gale, 2009, Bubble inclusion and removal using PDMS membrane-based gas permeation for applications in pumping, valving and mixing in microfluidic devices, J. Micromech. Microeng. 19095011.

24. J. Xu, R. Vaillant, D. Attinger, 2010, Use of a porous membrane for gas bubble removal in microfluidic channels: physical mechanisms and design criteria, Microfluidics and Nanofluidics Volume 9, Issue 4-5, pp 765-772.

25. X. Zhu, 2009, Micro/nanoporous membrane based gas-water separation in microchannel, Microsystem Technologies, Volume 15, Issue 9, pp 1459-1465.

26. J.H. Sung, M.L. Shuler, 2009, Prevention of air bubble formation in a microfluidic perfusion cell culture system using a microscale bubble trap, Biomedical Microdevices, Volume 11, Issue 4, pp 731-738.

27. D. Cheng, H. Jiang, 2009, A debubbler for microfluidics utilizing air-liquid interfaces, Applied Physics letters, 95, pp.214103.

28. K. Fei, T.S. Chen, C.W. Hong, 2010, Direct methanol fuel cell bubble transport simulations via thermal lattice Boltzmann and volume of fluid methods, Journal of Power Sources, Volume 195, Issue 7, pp 1940-1945.

29. S. Osher and J. A. Sethian, 1988, Fronts propagating with curvature dependent speed: Algorithms based on Hamilton-Jacobi formulation, J. Comput. Phys. 79, Issue 1, pp 12-49. 
30. P. Quan, B. Zhou, A. Sobiesiak, Z. Liu, 2005, Water behavior in serpentine micro-channel for proton exchange membrane fuel cell cathode, Journal of Power Sources, Volume 152, pp 131-145.

31. S. Kang, B. Zhou, 2014, Numerical study of bubble generation and transport in a serpentine channel with a T-junction, International Journal of Hydrogen Energy Volume 39, Issue 5, pp 2325-2333.

32. T. Taha, Z.F. Cui, 2006, CFD modelling of slug flow inside square capillaries, Chemical Engineering Science Volume 61, Issue 2, pp 665-675.

33. K. Fukagata, N. Kasagi, P. Ua-arayaporn, T. Himeno, 2007, Numerical simulation of gasliquid two-phase flow and convective heat transfer in a micro tube, International Journal of Heat and Fluid Flow, Volume 28, Issue 1, pp 72-82.

34. D. Gobby, P. Angeli and A. Gavriilidis, 2001, Mixing characteristics of T-type microfluidic mixers, J. Micromech. Microeng. Vol. 11, p 126.

35. R. Vundavilli and J. Darabi, Bubble Removal in Microfluidic Devices Using Nanofibrous Membranes, FEDSM2014-21616, Proceedings of the ASME 2014 4th Joint US-European Fluids Engineering Division Summer Meeting and 12th International Conference on Nanochannels, Microchannels, and Minichannels FEDSM2014 August 3-7, 2014, Chicago, Illinois, USA

36. C.N. Davies, 1952, The separation of the airborne dust and particles, Proceedings of Institute of Mechanical Engineers B1, London, pp 185-213.

37. M.M. Tomadakis, J.T. Robertson, 2005, Viscous permeability of random fiber structures: Comparison of electrical and diffusional estimates with experimental and analytical results, Journal of Composite Materials Volume 39, Issue 2, pp 163-188.

38. J.E. Drummond, M.I. Tahir, 1984, Laminar viscous flow through regular arrays of parallel solid cylinders, International Journal of Multiphase Flow Volume 10, Issue 3, pp 515540.

39. C.W. Hirt and B.D. Nichols, 1981, Volume of Fluid (VOF) Method for the Dynamics of Free Boundaries, Journal of Computational Physics 39, pp 201-225.

40. J.U. Brackbill, D.B. Kothe, C. Zemach, 1992, A continuum method for modeling surface tension, J of Computational Physics 100, pp 335-354. 
41. S. Jaganathana, H. Vahedi Tafreshib, B. Pourdeyhimia, 2008, A realistic approach for modeling permeability of fibrous media: 3-D imaging coupled with CFD simulation, Chemical Engineering Science Volume 63, Issue 1, pp 244-252.

42. H. Gholami Derami and J. Darabi, Computational and experimental study of gas bubbles removal in a microfluidic system, ASME 2015 12th International Conference on Nanochannels, Microchannels, and Minichannels, July 6-9, 2015, San Francisco, California, USA. 